\title{
marges Marges
}

revue d'art contemporain Revue d'art contemporain

19 | 2014

Les temps de l'art

\section{Le « silence-après » d'Yves Klein (1958-1961)}

The "after-silence" of Yves Klein

\section{Noémi Joly}

\section{(2) OpenEdition}

\section{Journals}

Édition électronique

URL : http://journals.openedition.org/marges/991

DOI : 10.4000/marges.991

ISSN : 2416-8742

\section{Éditeur}

Presses universitaires de Vincennes

\section{Édition imprimée}

Date de publication : 1 octobre 2014

Pagination : 74-84

ISBN : 978-2-84292-415-7

ISSN : $1767-7114$

Référence électronique

Noémi Joly, «Le « silence-après » d'Yves Klein (1958-1961) », Marges [En ligne], 19 | 2014, mis en ligne le 01 octobre 2016, consulté le 03 mai 2019. URL : http://journals.openedition.org/marges/991 ; DOI : 10.4000/marges.991 


\section{Le «silence-après » d'Yves Klein (1958-1961)}

/1 Nous employons de manière générique l'expression Symphonie Monoton pour désigner les différentes versions de ce projet entre 1958 et 1961. C'est ainsi qu'Yves Klein s'y réfèrait, dans ses écrits comme dans ses discours, jusqu'en 1961, date à laquelle il arrêta d'employer le titre de Symphonie Monoton-Silence. Ce titre est celui inscrit sur la partition écrite par Louis Saguer.
Le corpus sonore et musical de l'œuvre d'Yves Klein se résume à sa célèbre Symphonie Monoton/1 et à son paratexte, dont l'apparente évidence - la monotonie comme équivalent conceptuel et sonore de la monochromie picturale - aura sans doute découragé les tentatives d'études plus approfondies. Et pourtant, si un tableau monochrome d'Yves Klein se caractérise bien par une couleur uniformément appliquée sur une toile, sans nuance de touche ni de valeur, la monotonie ne se laisse pas si aisément caractériser dans ses manifestations audibles. Le déploiement diachronique de cette création musicale eut pour effet sa reconfiguration en de multiples immanences, des premières sonorités électroniques (vers 1958-1959) à la mise en évidence du silence (1960-1961), en passant par son instrumentation pour chœur et orchestre de chambre lors de sa première présentation publique (1960). La Symphonie Monoton ne se contentait pas d'infiltrer la frontière rendue poreuse entre arts de l'espace et du temps. Ses principales modifications accompagnèrent les inflexions théoriques d'Yves Klein mais aussi ses déplacements géographiques. Le récit que nous nous proposons de retracer de l'élaboration de cette 
œuvre met en avant le jeu d'échanges, immédiats ou temporisés, qui se tissèrent avec ses contemporains (Pierre Henry, Karlheinz Stockhausen, John Cage, Louis Saguer), de Paris à New York via Cologne et Gelsenkirchen. La temporalité active des échanges réciproques et des « influences » - soit les processus d'interprétation, d'appropriation idiosyncratique, d'une pensée flottante, telle que le "silence " de John Cage - contribua à la dilatation du temps de la création et à la constante ré-élaboration de la Symphonie Monoton.

\section{Paris, printemps 1958 : une collaboration inachevée avec Pierre Henry}

Si ce projet remonte à 1947 ou 1949, selon les dires d'Yves Klein, les premiers sons furent prélevés, collectés, par Pierre Henry, figure tutélaire de la musique concrète, dont Klein s'attacha la compétence au printemps 1958, au moment de l'exposition dite du «Vide », à la galerie Iris Clert/2. Alors que le monochrome était pour Yves Klein le support d'une qualité ineffable, essentielle, désignée comme «sensibilité picturale immatérielle », il envisagea, avec l'exposition dite du "Vide », de dissocier la sensibilité picturale de son support habituel (tableau), afin de la présenter directement, sans aucune médiation: «Il n'y a à présent plus d'intermédiaire: on se trouve littéralement imprégné par l'état sensible pictural spécialisé et stabilisé au préalable par le peintre/3... ». C'est dans ce contexte que Pierre Henry fut chargé de s'atteler à la Symphonie Monoton d'Yves Klein/4.

La rencontre entre Pierre Henry et Yves Klein avait eu lieu à la fin de l'année 1956, par l'intermédiaire de Claude Pascal/5, poète et ami de Klein, pour le compte duquel Pierre Henry avait déjà mis en musique quelques poèmes (L'An bleu et L'Occident est bleu). L'intérêt commun du compositeur et de l'artiste pour le lettriste François Dufrêne confirma de surcroît le rapprochement entre les deux hommes: en mai 1957, Yves Klein, électron libre gravitant autour du lettrisme, avait en effet procédé à l'enregistrement d'un cri éructé par François Dufrêne, un «cri bleu » sans modulation, qui se développait de manière platement récitative. Cette tentative, sans lendemain dans ses modalités, de transposer vocalement la monochromie faisait écho à la tirade débitée puis vociférée par un speaker allemand, que Pierre Henry avait intégré dans son Voile d'Orphée (1953). Le compositeur reconnaissait d'ailleurs volontiers dans la formation de la texture sonore de son imaginaire musical, l'importance du « langage écrit et verbal, [du] cri, [de] François Dufrêne/6 ".

Toutefois, les sons que Pierre Henry entreprit de collecter et d'inventer en vue de de la réalisation de la Symphonie de Klein s'écartaient
/ 2 Yves Klein, «La Spécialisation de la sensibilité à l'état matière première en sensibilité picturale stabilisée », exposition dite du "Vide ", Paris, Galerie Iris Clert, 28 avril-5 mai 1958.

/3 Yves Klein, «Le dépassement de la problématique de l'art » (1959), dans Yves Klein, Le Dépassement de la problématique de l'art et autres écrits, Paris, ENSBA, coll. " écrits d'artistes ", 2003, p. 84. Cette anthologie des écrits de Klein sera désormais désignée par l'abréviation Écrits.

/4 Pierre Henry, "Une ouverture» [propos recueillis en 1982], dans Jean-Yves Mock (sld), Yves Klein, catalogue d'exposition (Paris, MNAM, Centre Georges Pompidou, 1983), Paris, Centre Georges Pompidou, 1983, p. 265.

/5 Claude Pascal et Pierre Henry s'étaient quant à eux rencontrés grâce à Eliane Radigue, élève de Pierre Schaeffer et assistante de Pierre Henry, alors mariée avec Arman, le troisième membre de ce trio d'amis niçois.

/6 Pierre Henry, op. cit., p. 265. 
/8 Michel Chion, La Musique concrète, art des sons fixés, Lyon, Mômeludies éditions, 2009.

/9 Yves Klein, "L'évolution de l'art vers l'immatériel. Conférence à la Sorbonne » [3 juin 1959], dans Écrits, op. cit., p. 120-153.

/10 ibid., p. 143-144. ostensiblement de l'option monovocale des Cris bleus: "[...] les sons existaient absolument. On peut encore les lire dans mes cahiers de classement. Des sons filés, des sons scintillants, des pulsations. Tout ça pour lui, pour ses idées/7 ». La démarche que décrit Pierre Henry se présente comme un travail de recherche de matériaux sonores, qu'il nomme et indexe ensuite dans ses cahiers de classement, véritables répertoires de sa sonothèque borgésienne. Ces sons procédaient de prélèvements sur des disques enregistrés, de brèves saisies arrachées à une continuité musicale et ensuite retravaillées. Pareille démarche s'inscrit en somme dans la révolution de la musique concrète, «l'art de manier le son fixé/8 ». Si le son pouvait être de source variée, Klein le préféra d'emblée électronique et le choisit de la sorte pour l'extrait de la première version de la Symphonie qu'il donna à écouter, une sorte d'accroche diffusée lors de sa conférence à la Sorbonne du 3 juin 1959, dont le sujet portait sur « L'évolution de l'art vers l'immatériel/9 ». À l'écoute, cet extrait de sa Symphonie consistait alors en un son unique - littéralement un «monoton » - électronique, dont l'attaque et la chute avaient été amputées, un son plat, tenu, monotone, sans rythme ni mélodie.

La dénomination de "symphonie » arrimée à un projet musical aussi minimal répercutait l'emprise du dialogue avec Pierre Henry et résonnait comme un hommage à la Symphonie pour un homme seul de ce dernier, créée en 1951 et mise en ballet par le chorégraphe Maurice Béjart en 1955. Quant à sa forme même, elle répondait au projet qu'énonça Yves Klein en ces termes, dans sa conférence à la Sorbonne: "Cet extrait de ma Symphonie Monoton que vous avez écouté au début est un son électronique. La symphonie à l'origine durait quarante minutes. Elle était aussi longue justement pour montrer le désir de vaincre le temps. L'attaque et la fin de ce son avaient été coupées, ce qui provoquait une sorte de phénomène sonore étrange aspirant la sensibilité. En effet, n'ayant ni commencement ni fin, même imperceptibles, cette symphonie sortait de la phénoménologie du temps, devenait extérieure au passé, au présent, au futur puisqu'elle n'était jamais née ni morte en somme, après existence cependant dans la réalité sonore physique/10. ». Le parti pris anti-compositionnel de cette Symphonie sans début ni fin n'était donc pas explicité comme l'adaptation musicale (temporelle) d'une œuvre picturale (spatiale) mais témoignait d'un paradoxal « désir de vaincre le temps ». Le temps phénoménologique ou temps de l'expérience vécue, celui qui nous affecte, comme le temps historique, qui articule les trois modes passé-présent-futur, étaient rejetés en bloc au profit de la conception d'un présent étiré, dilaté, que rendait sensible l'extrait sonore et monotone. 
Cette volonté affichée d'affranchir son art du temps historique et, plus particulièrement, du régime d'historicité de la modernité caractérisée par un tropisme futuriste, avait pour corollaire une exaltation du temps cosmique, dont la magnitude temporelle affole tout repère anthropocentré. Pour Yves Klein, il ne s'agissait pas de mettre son art au diapason de la frénésie de l'époque, mais d'établir une harmonie cosmique avec l'univers: «Mon art n'appartiendra pas à l'époque, pas plus que l'art de tous les grands classiques n'a appartenu aux époques où ils ont vécu, parce que je recherche avant tout, comme eux, à créer dans mes réalisations cette "transparence", ce "vide" incommensurable dans lequel vit l'esprit permanent et absolu délivré de toutes dimensions/11. ». Et lorsque, à propos de cette Symphonie Monoton, il déclarait que le thème était sa vie/12, il fallait comprendre que la vie, telle que l'envisageait Yves Klein, était appréhendée comme une forme de présent absolu, extra-temporel, délié du présent "phénoménologique » comme historique. Par cette sacralisation de la vie, Klein professait une communion mystique avec l'univers adimensionnel et cosmique. Là encore, le discours d'Yves Klein et la pensée de Pierre Henry se rejoignaient et attestaient que la rencontre, féconde, reposait sur une véritable affinité spirituelle/13. Comme pour approfondir leur dialogue, Pierre Henry avait en effet communiqué à son collaborateur un texte daté de février 1947 et programmatiquement intitulé "Pour penser à une nouvelle musique » : "Il faut détruire la musique [...]. Il faut prendre immédiatement une direction qui mène à l'organique pur. À ce point de vue, la musique a été beaucoup moins loin que la poésie ou la peinture. Elle n'a pas encore osé se détruire elle-même pour vivre. Pour vivre plus fort comme le fait tout phénomène vraiment vivant [...]. Le mythe du moderne n'existe plus. Les bruits seront supprimés, désignifiés et comme désacralisés. Alors, ce sera peut-être la musique concrète, la musique du vivant et du soleil/14. ». Les déclarations d'Yves Klein font indéniablement écho à l'exaltation, au lyrisme qui affleurent dans ces quelques lignes de Pierre Henry où «le mythe du moderne » est anéanti pour céder les devants à « la musique concrète, la musique du vivant et du soleil »
/11 Yves Klein, « Théâtre du Vide ", dimanche 27 novembre 1960 (Le journal d'un seul jour) [1960], dans Écrits, op.cit, p. 176.

/12 Yves Klein: «Pendant cette période de condensation, je crée vers 1947-1948 une symphonie monoton dont le thème est ce que je voulais que soit ma vie ». Dans Yves Klein, "Le dépassement..." ", op. cit., p. 82.

/13 Sur la réciprocité de l'échange entre Pierre Henry et Yves Klein, voir Pierre Henry, op. cit., p. $265-266$.

/14 Pierre Henry, "Pour penser à une nouvelle musique» (1950), dans Pierre Henry, Journal de mes sons (1979), suivi de Préfaces et Manifestes, Arles, Actes Sud, 2004, p. 99-101.

/15 Durée de 37' musique électronique. Changement d'appellation opéré en 2000, à la demande de Madame Yves Klein. Voir le site de l'IRCAM [http://brahms.ircam. $\mathrm{fr} /$ works/work/22173/], consulté le 28 septembre 2013.

\section{Gelsenkirchen, 1959 : l'immédiat retour aux codes musicaux}

Et pourtant, la collaboration n'excéda pas le stade du dialogue et de la recherche. La Symphonie Monoton $\mathrm{n}^{\circ} 2$, récemment rebaptisée Monochromie/15, que Pierre Henry offrit à Yves Klein en cadeau de mariage en 1962, était un post-scriptum tardif de leurs recherches, 
déjà obsolète par rapport au tour qu'avait pris la démarche de Klein. Le tournant eut lieu lors du séjour de Klein dans la Ruhr, à Gelsenkirchen, entre octobre 1958 et juin 1959, dans le cadre de la réalisation d'un décor monumental pour l'opéra de la ville.

L'échelle du lieu, la nature du programme (un Musiktheater), l'ampleur de la commande, semblent avoir eu raison des premières orientations de Klein et Henry. Tout se passe comme si le travail de titan accompli par Klein en ce lieu avait contribué à redonner toute sa puissance au musical, au sens le plus conventionnel du terme. Pareille grandeur devait en effet s'accompagner d'un retour marqué aux codes mucaux: performance et partition. Les photographies prises par Charles Wilp montrent Klein en chef d'orchestre autoritaire, au regard hypnotique, dirigeant énergiquement sa symphonie devant un orchestre et un parterre vides.

À son retour d'Allemagne, cette attitude cérémonieuse s'accentua encore avec l'organisation d'une séance d'Anthropométries de l'époque bleue, le 9 mars 1960, à la Galerie internationale d'Art contemporain, de Paris: un orchestre de chambre formé de six instrumentistes (trois violons, trois violoncelles) et accompagné au chant par trois choristes assurait le contrepoint musical de la performance. La première représentation publique de la Symphonie, celle du 9 mars 1960, est donc antérieure à l'écriture des deux partitions, datées de 1961, qui furent commandées par Yves Klein au compositeur Louis Saguer, au moment de la rétrospective "Yves Klein, Monochrome und Feuer", du Museum Haus Lange de Krefeld (14 janvier - 26 février 1961). La partition fut ensuite interprétée par Philippe Arrii-Blachette en présence d'Yves Klein. En d'autres termes, la partition de Louis Saguer, c'est-à-dire la notation abstraite des sons, visait à délimiter un cadre pour l'interprétation et rompait ainsi définitivement avec la musique concrète. Rappelons que, par définition, aucun intermédiaire écrit, aucune partition, ne s'interposent entre la production et l'audition dans la musique concrète, d'où il résulte que la musique concrète se passe naturellement d'interprètes.

Le dernier plan que dessina Klein pour son exposition de Krefeld de janvier 1961 réservait la plus vaste salle du musée à l'orchestre disposé en hémicycle et y prévoyait un accrochage composé de monochromes bleus et de Reliefs éponges. Yves Klein établissait par là même une étroite correspondance entre la monochromie et sa symphonie. Davantage, cette immense salle ordonnait stratégiquement le parcours de l'exposition et, vue en plan, se présentait comme une sorte d'immense nef attenante à un sanctuaire dédié au "Vide ". Cette symbolique spatiale semblait confirmée par la présence, dans la salle antérieure, d'une Sculpture éponge, que Klein considérait 
comme un portrait-robot de son spectateur idéal, imprégné et bleui par la sensibilité immatérielle volatile, que donnaient à voir et à entendre monochromie et monotonie. Comme si le cumul des sollicitations sensorielles était à même de catalyser la transformation spirituelle du spectateur. La configuration des espaces scénographiait le processus d'une initiation quasi-rituelle du spectateur, autorisé à communier avec et dans le Vide seulement après une phase préliminaire d’imprégnation physique dans le vaisseau central.

\section{Cologne, 1959 : l'échec d'un environnement sonore avec Karlheinz Stockhausen}

Lorsque Klein arriva en Allemagne, sur le chantier de l'opéra de Gelsenkirchen, une véritable effervescence intellectuelle entourait la présence de John Cage et de Karlheinz Stockhausen. Klein rencontra Stockhausen à son domicile à Cologne, en 1959. Né en 1927, de la même génération qu'Yves Klein (1928-1962) et que Pierre Henry (1927), Stockhausen avait suivi les cours d'analyse musicale d'Olivier Messiaen à Paris et, après un très bref passage au studio de Pierre Schaeffer à l'ORTF, où il s'était essayé à la pratique du ciseau, il s'était orienté vers le champ de la musique électronique. Il travaillait aussi depuis 1953 au Studio de musique électronique de la WDR (Westdeutscher Rundfunk) de Cologne, fondé par Herbert Eimert. Le Studio de musique électronique de Cologne passait alors pour être l'équivalent du GRM (Groupe de Recherche Musicale) intégré à l'ORTF, où officiaient Pierre Schaeffer et Pierre Henry (ce dernier jusque 1959). Lorsque Klein, par l'entremise de son galeriste Alfred Schmela, prit contact avec Stockhausen, il s'attendait, non sans raison, à rencontrer l'alter ego allemand de Pierre Schaeffer. Cependant, dès 1953-1954, dans un contexte de rivalités marquées, un clivage s'était opéré entre musique concrète française et musique électronique allemande, l'une et l'autre démarche étant distinguée par ses sources sonores, c'està-dire par la façon de générer les sons. Aux concrets étaient réservés les sons prélevés par microphone; aux autres, les sons de synthèse. Ainsi en avait-il été de la première œuvre de musique électronique pour sons sinusoïdaux de Stockhausen, Studie I, composée en 1953 et réalisée par un générateur de fréquences.

Outre ses innovations concernant le son électronique, Stockhausen attirait alors l'attention pour ce qu'il avait entrepris en matière de spatialisation du son, tant sur le plan de la réalisation que de la théorie. Avec Gruppen, créé le 24 mars 1958 par l'Orchestre symphonique de la Radio de Cologne/16, l'occupation de l'espace devenait une composante à part entière de l'œuvre et impliquait l'auditeur dans une
/16 La première eut lieu dans la Rheinsaal de la Foire de Cologne et fut retransmise dans le programme "Musik der Zeit » de la WDR. 
/17 Conférence reprise dans Dieter Schnebel (sld), Texte zur elektronischen und instrumentalen Musik, vol.1, Cologne, DuMont Schauberg, 1963, p. 152-175.

\section{/18 Karlheinz}

Stockhausen, «Invention et découverte », Cahiers Renaud-Barrault, "La Musique et ses problèmes contemporains ", Paris, Julliard, 1963, p. 163.

/19 Pierre Henry, Journal de mes sons, op. cit.

\begin{abstract}
/20 Yves Klein, "Sensibilité pure », dans Écrits, op. cit., p. 182.
\end{abstract}

/ 21 Lettres d'Yves Klein à Werner et Anita Ruhnau, datées du 18 mars et du 18 juin 1958. La première visite de Klein eut lieu avant l'inauguration, grâce à sa compagne d'alors, l'architecte Bernadette Allain et on peut supposer que sa curiosité architecturale en fut renforcée.

/ 22 Le 2 mai 1958 y fut créé le Poème électronique d'Edgar Varèse, composition électronique pour bande magnétique à trois pistes, diffusée sur 425 haut-parleurs. Durée: 8 minutes 5 secondes. [http://brahms.ircam. $\mathrm{fr} /$ works/work/12512/], consulté le 28 septembre 2013. écoute multidirectionnelle. L'orchestre de neuf musiciens avait été dispersé en trois orchestres distincts et homogènes; disposées en fer à cheval en face, à gauche, à droite de l'auditeur, les trois sources sonores, respectivement dirigées par Karlheinz Stockhausen, Bruno Maderna et Pierre Boulez, étaient ainsi entendues simultanément. Quelques mois plus tard, le 4 septembre 1958 , lors d'une session des Cours d'été de Darmstadt (Internationale Ferienkurse für neue Musik), Stockhausen avait tenu une conférence sur la «Musique dans l'espace » [Musik im Raum], première formulation théorique de son expérimentation spatiale, conférence qui fut reprise à Bruxelles en octobre 1958 lors des "Journées de musique expérimentale »/17. L'espace s'imposait ainsi comme un paramètre structurant de l'œuvre musicale, ce dont il s'expliqua avec clarté et concision quelques temps plus tard: «Ici, je veux à nouveau mentionner la redécouverte de la fonction spatiale en ce qui concerne la composition musicale. Direction et forme du mouvement des sons dans l'espace sont d'une importance aussi grande que toutes les qualités sonores/18 $»$.

Or, Pierre Henry, dès l'époque du «Vide » et pour répondre aux aspirations d'Yves Klein, avait initié une réflexion sur l'environnement sonore: "Tout cela m'[avait] ouvert une nouvelle voie : "environner" la musique/19. ». L'environnement sonore que recherchait Yves Klein était une densité sonore, une amplitude spatiale du son, analogue à la sensibilité immatérielle qui emplissait l'espace du «Vide » et dont le fantasme innervait également d'autres projets non réalisés. Ainsi, dans le texte "Sensibilité pure » publié dans dimanche 27 novembre 1960 (le journal d'un seul jour), en 1960, Klein recensait le projet suivant: "Le rideau se lève lentement avec l'éclosion d'un pétillement continu, semblable à celui que fait l'eau fraîche gazeuse débouchée, mais prodigieusement amplifié. C'est une inondation sonore, monotone, s'imprégnant d'une manière volumétrique dans l'espace, perceptible par l'ouïe sensible de chaque spectateur/20 ». Enfin, cette problématique de la spatialisation du son dont s'emparaient musiciens et architectes était dans l'air du temps. Lorsque Klein, sur le chemin de Gelsenkirchen, visita, à deux reprises au moins, l'Exposition universelle de Bruxelles de 1958/21, il ne put que remarquer le Pavillon Philips, conçu par Le Corbusier et lannis Xenakis, et qui proposait une alternative inédite à l'architecture conventionnelle des lieux de diffusion et d'écoute de la musique, créant les conditions architecturales d'une véritable projection de la musique dans l'espace/22.

Pourtant, si la quête d'un environnement sonore pour sa Symphonie conduisit Yves Klein à entrer en contact avec Karlheinz Stockhausen, précisément parce que le compositeur passait alors pour être l'un des pionniers dans le domaine de la spatialisation physique de 
l'œuvre musicale, la collaboration ne se fit pas. Selon l'assistant de Klein à l'époque, Jean-Pierre Mirouze, la raison en fut que Stockhausen était trop avancé dans ses recherches pour accepter cette coopération/23. Le récit de cette rencontre est donc celui d'un échec, d'une collaboration avortée.

\section{Düsseldorf, automne 1958, New York, avril 1961: la réception différée du silence de John Cage}

Il est vraisemblable qu'Alfred Schmela, le galeriste d'Yves Klein, intervint pour créer les conditions de la rencontre entre Stockhausen et son protégé. II n'est pas interdit de penser que Schmela ait ainsi cherché à s'attirer les faveurs de Stockhausen afin de faire contrepoids à la présence de John Cage dans l'autre espace d'avant-garde de Düsseldorf, la galerie 22, de son collègue Jean-Pierre Wilhelm. Les deux lieux avaient en effet ouvert à quelques semaines d'intervalle à Düsseldorf, en mai 1957. Aucune rencontre entre Klein et Cage n'est attestée et elle n'eut sans doute pas lieu, même si Klein, dans la première mouture de son "Centre de la Sensibilité " pressentait John Cage comme "professeur de théâtre/24 " [sic]. Cependant, la présence de Cage à Cologne et Düsseldorf lors de sa tournée européenne en 1958 coïncidait avec les dates du séjour de Klein à Gelsenkirchen et on présume que Klein, grand incubateur, fut prompt à s'emparer de tout ce que le contexte désignait à son attention pour approfondir sa pensée. Le séjour en Allemagne de Klein fut donc l'occasion d'une première rencontre avec la pensée de Cage, d'une incubation de ses idées, dont l'effet fut temporisé dans le discours et l'œuvre musicale de Klein: le révélateur ne se produisit que deux ans plus tard, en 1961, aux États-Unis.

L'intérêt voué à John Cage prend communément appui sur les deux notions clés du silence et de l'indétermination. L'une et l'autre furent abondamment commentées dès sa tournée européenne de 1958, dont les principales haltes à l'automne 1958 furent successivement: les Internationale Ferienkurse für neue Musik de Darmstadt, où il tint trois conférences-récitals les 6, 8 et 9 septembre; le Centre de musique contemporaine de la WDR à Cologne, qui englobait également le studio électronique de Stockhausen, où eut lieu la première européenne de son Concert for Piano and Orchestra le 19 septembre; les " Journées internationales de musique expérimentale » du 5 au 10 octobre 1958, qui se tinrent dans le cadre de l'Exposition de Bruxelles et lors desquelles Cage prononça sa célèbre conférence sur l'indétermination (« Indeterminacy: New Aspect of Form in Instrumental and Electronic Music »). Après avoir assisté au concert de Cologne et avoir
/ 23 Jean-Pierre Mirouze, cité dans un entretien avec Marion Guibert de février 2006, dans Camille Morineau (sld), Yves Klein.

Corps, couleur, immatériel, catalogue d'exposition (Paris, Centre Pompidou, 2006), Paris, Centre Pompidou, 2006, p. 276.

/ 24 Dans «Le Dépassement de la problématique de l'art", dans Écrits, op. cit., p. 115 : sous la rubrique, " théâtre ", Klein suggère les professeurs suivants: Claude Pascal, Pierre Henry, Sylvano Bussoti, Mauricio Kagel, Jacques Polieri. Cependant, dans le texte établi en allemand avec Werner Ruhnau, John Cage remplaçait Claude Pascal au même poste. 
/25 Rétrospective «Dada: Dokumente einer Bewegung », Düsseldorf, Kunsthalle, 5 septembre-19 octobre 1958.

/ 26 Il convient ici de mentionner qu'Yves Klein avait assisté le 30 juin 1952 à l'avant-première des Hurlements en faveur de Sade de Guy Debord, au ciné-club Avant-Garde 52, film qui s'achevait sur une séquence de 24 'privée de tout son comme de toute image. Sur les relations ambiguës entre Guy Debord et Yves Klein, nous renvoyons le lecteur aux travaux de Kaira M. Cabañas. Kaira M. Cabañas, « Yves Klein en France: un paradoxe spatial ", dans Camille Morineau (sld), Yves Klein. Corps, couleur, immatériel, op. cit., p. 174-181.; Kaira M. Cabañas, The Myth of Nouveau Réalisme: art and the performative in postwar France, New Haven, Londres, Yale University Press, 2013.

/ 27 John Cage, «Composition as processes. III. Communication ", dans John Cage, Silence, conférences et écrits [1961], trad. V. Barras, Genève, Héros-Limite, Contrechamps, 2003.

/28 Yves Klein, « L'aventure monochrome ", dans Écrits, op. cit., p. 232. visité la rétrospective "Dada » à la Kunsthalle de Düsseldorf/25 avec John Cage, le galeriste Jean-Pierre Wilhelm décida de jouer les prolongations dans sa galerie de Düsseldorf et y programma Music Walk, le 14 octobre, avec John Cage en personne, David Tudor et Cornelius Cardew. Le livre d'or de sa galerie, qui recense les signatures du cercle de compositeurs du studio de la WDR de Cologne et des participants des cours de Darmstadt, atteste que sa galerie était alors un véritable carrefour des sociabilités musicale et artistique. Autant de faits qui rappellent la présence remarquée de John Cage dans la région, au moment où Klein s'installait à Gelsenkirchen.

Plutôt que d'en inférer une influence passive, subie, de Cage sur Klein, nous alléguons que Klein procéda à une opération de sélection, d'appropriation dynamique, des idées de son aîné. Car pour l'artiste, tout n'était pas bon à prendre chez Cage : Klein demeura ainsi hermétique au hasard et à l'indétermination, qui agitaient pourtant beaucoup la communauté musicale. Selon Cage, les opérations de hasard découlaient de l'abandon de toute intentionnalité, ce qui était passablement étranger à Klein, méfiant envers tout ce qui pouvait le compromettre avec l'esprit dada. Klein fut en revanche bien plus réceptif à la question du silence/26. D'abord parce que Cage l'avait abordée en lien avec l'écoute: « Pourquoi y a-t-il tant de gens qui ont du mal à écouter? Pourquoi se mettent-ils à parler dès qu'il y a quelque chose à entendre? Ont-ils les oreilles non pas de chaque côté de la tête mais situées à l'intérieur de la bouche, si bien que quand ils entendent quelque chose, leur première réaction est de parler/27? ", interrogeait John Cage. Cette stigmatisation des comportements s'accordait avec la dénonciation récurrente, dans les écrits de Klein, de l'aveuglement des spectateurs désemparés devant ses monochromes car «prisonniers de l'optique apprise/28 ». Pour Cage, comme pour Klein, leurs œuvres exigeaient de lâcher les armes, de se mettre dans un état de disponibilité, de participation active, sinon d'empathie.

Ensuite parce que, grâce à John Cage, le silence était en passe de devenir une matière sonore de plein droit. Au sujet du très célèbre 4'33", Tacet for Any Instruments, dont la première avait été jouée par David Tudor le 29 août 1952, à Woodstock puis redonnée à Darmstadt en 1958, David Tudor rapporta qu'il s'agissait d'une œuvre en trois mouvements: dès qu'il prit place, la météorologie du jour avait voulu que le premier fut celui du vent; que le second fut celui de la pluie crépitant sur la verrière. Quant au troisième, il fut celui de l'assistance déconcertée ou ravie. Par cette œuvre, Cage portait à la conscience de chacun que le silence véritable n'existait pas et que la tranche de durée arbitrairement définie par 4'33" n'était qu'un "filet jeté au hasard ", qui captait les sons ambiants comme ils venaient/29. » En somme, le 
silence de Cage était aussi bruyant que le vide de Klein était saturé. De même que Cage avait décidé de faire taire la musique pour que le son émerge, Klein, avec l'exposition du «Vide », avait congédié les tableaux pour laisser advenir l'immatériel.

Enfin, concernant la Symphonie Monoton - qui, rappelons-le, est intrinsèquement liée au «Vide » dans sa genèse même - c'est dans Le Dépassement de la problématique de l'art, rédigé par Klein au cours de l'année 1959, que la question du silence commença à poindre: «Cette symphonie n'existe donc pas tout en étant là, parce qu'elle n'est jamais née ni morte, après son existence cependant, dans le monde de nos possibilités de perception conscientes: c'est $d u$ silence, présence audible/30. ". La réflexion sur le silence s'affirma surtout autour de 1960-1961, d'une part dans les écrits de Klein et, d'autre part, dans la Partition de la Symphonie Monoton - Silence 1947-1961, dont l'annotation manuscrite précisait: "Durée de 5 à 7 minutes plus 44 secondes de silence absolu ». La mention d'une durée déclinée en minutes et en secondes et décidée en amont s'apparentait déjà à une citation de la partition de son aîné et entraînait corrélativement un nouveau rapport au chef d'orchestre, manifeste dans la représentation du 9 mars 1960, très proche de celui qu'avait énoncé Cage dans une conférence de 1958: "Le chef d’orchestre n'est plus un policier, c'est simplement un indicateur de temps - non en battues - comme un chronomètre/31. ".

Le différé temporel de la réception de Cage par Klein est particulièrement manifeste dans deux textes qui renchérissent sur le silence et l'érigent en phénomène sonore structurant de sa symphonie. Le premier, titré « Yves Le Monochrome 1960. Le Vrai devient réalité », avait été spécialement rédigé par Yves Klein pour le troisième et dernier numéro de la revue ZERO. Le second fut le Manifeste de l'Hôtel Chelsea, écrit au moment du séjour de l'artiste à New York au printemps 1961/32, adressé à un public américain plus au fait des White Paintings de Robert Rauschenberg et de la musique de Cage que de la monochromie européenne. Comme sur la partition, la symphonie était opportunément rebaptisée: Monotone - Silence - Symphonie. Comme l'a souligné Harold Bloom dans L'Angoisse de l'Influence/33, toute compréhension active, authentique, est une espèce de dialogue. Yves Klein avait engagé un dialogue polémique avec le silence de John Cage dont le Manifeste de l'Hôtel Chelsea constitua l'énonciation. Il ne s'agissait donc aucunement d'une reprise mimétique dans la mesure où le "silence-après » de Klein s'interprète bien comme une misprision, c'est-à-dire comme une incompréhension intentionnelle. Significativement, ce n'était plus le son unique, ni le son électronique, ni la création d'un continuum sonore et spatial,
/29 « Mon œuvre a pour but de faire la démonstration de la vie. La vie se passe très bien en moi, et cela vous expliquera ma pièce silencieuse, 4' 33" ". Richard Kostelanetz, Conversations avec John Cage, trad. M. Dachy, M. Fong et M. Guénot-Hovnanian, Paris, Éd. des Syrtes, 2000.

/30 Yves Klein, "Le Dépassement... », dans Écrits, op. cit., p. 82 (nous soulignons). Nous renvoyons le lecteur à la parution récente du catalogue Audible Presence. Lucio Fontana, Yves Klein, Cy Twombly (New York, Galerie Dominique Levy, 2013), New York, Dominique Lévy, 2013.

/31 John Cage, Silence. Lectures and Writings, 1973, Middletown, Wesleyan University Press, p. 72.

/32 Yves Klein, "Manifeste de l'hôtel Chelsea » [1961], dans Écrits, op. cit., p. 303 .

/ 33 Harold Bloom, Théorie de l'influence [The Anxiety of Influence: A Theory of Poetry, New York: Oxford University Press, 1973], trad. M. Shelledy et S. Degachi, Paris, Éditions Aux forges de Vulcain, juin 2013. 
/34 « Yves

Le Monochrome 1960. Le Vrai devient réalité » (1960), dans Écrits, op. cit., p. 285.

/35 « ... c'est alors seulement que l'extraordinaire silence a posteriori... engendrera une nouvelle et unique zone de sensibilité picturale de l'immatériel ", dans Yves Klein, «Manifeste de l'hôtel Chelsea » (1961), op. cit., p. 303.

/36 Yves Klein, « Yves Le Monochrome 1960. Le Vrai devient réalité ", op. cit., p. 285.

/37 Yves Klein, "Manifeste de l'hôtel Chelsea ", op. cit., p. 303.

/38 Le mot mis entre guillemets par Yves Klein lui-même est un anglicisme démarqué du terme chance (hasard), souvent utilisé par John Cage.

/39 Yves Klein, «Yves Le Monochrome 1960. Le Vrai devient réalité », op. cit., p. 285 . qui caractérisaient désormais sa Symphonie mais la création d'un silence, d'un "silence-après " : "Le "silence-après" : après que tout fut terminé, dans chacun de nous tous, présents à cette manifestation. Le silence... c'est cela même ma symphonie, et non le son luimême, d'avant-pendant l'exécution/34. ॥.

L'histoire de la Symphonie Monoton d'Yves Klein met en évidence un processus de création lentement mûri, jalonné de phases de cristallisation. Le projet fut en effet remodelé à plusieurs reprises, dans un dialogue approfondi, amorcé ou indirect avec ses interlocuteurs, respectivement Pierre Henry, Karlheinz Stockhausen, Louis Saguer et John Cage. À l'intérieur de ce cadre général, une temporalité connexe s'est tramée, celle qui conduisit Yves Klein à l'appropriation et à la reformulation de la question du silence cagien. La réponse, différée, de Klein fut double: théorique d'une part avec la parution d'un manifeste et d'un article; concrète de l'autre, puisqu'elle s'incarna dans l'ultime révision de sa «symphonie », baptisée Symphonie Monoton-Silence, légitimant ainsi la part octroyée au silence non compositionnel dans la musique. Considéré à l'égal du son intentionnel et organisé, en l'occurrence du son continu de la première partie de la Symphonie, le silence était symboliquement valorisé comme générateur d'un espace ineffable et audible, d'une «zone de sensibilité picturale immatérielle/35 ». Et pourtant, ce silence qualifié d'« absolu » par Klein se démarquait du silence relatif, ouvert à tous les bruits environnants, de son aîné. Car le « silence-après » d'Yves Klein était, en dernière instance, un silence intériorisé, « dans chacun de nous tous/36 », « un pur silence affectif/37 ». Face à ce qu'il percevait peut-être comme une banalisation dadaïste de l'art, et de la musique en particulier, le silence de la Symphonie s'apparentait à une révélation ambiguë, à la fois ancrée dans le présent et déliée de tout arrimage temporel: « C'est ce silence si merveilleux qui donne la "chance"/38 et qui donne même parfois la possibilité d'être vraiment heureux, ne serait-ce qu'un seul instant, pendant un instant incommensurable en durée/39. ».

\section{Noémi Joly}

